\title{
Características epidemiológicas da contaminação por COVID-19 no estado da Bahia
}

\author{
Epidemiological characteristics of COVID-19 \\ contamination in the state of Bahia
}

\author{
Aline Gonçalves Machado' (1) \\ Mayara dos Santos Batista2 (1) \\ Marcio Costa de Souza ${ }^{3}$ (1)
}

${ }^{1}$ Autora para correspondência. Universidade do Estado da Bahia (Salvador). Bahia, Brasil. linnemachs@gmail.com

2-3Universidade do Estado da Bahia (Salvador). Bahia, Brasil. msbatissta@gmail.com, mcsouza@uneb.br

RESUMO | OBJETIVO: Caracterizar o perfil epidemiológico dos casos confirmados e óbitos por COVID-19 no estado da Bahia. MÉTODOS: Estudo epidemiológico descritivo e retrospectivo realizado a partir de dados secundários extraídos do Boletim Epidemiológico do Estado da Bahia apresentados em forma de tabela com números absolutos e relativos. RESULTADOS: Foi identificado que a maioria dos casos confirmados são indivíduos do sexo feminino numa faixa etária de 30 a 39 anos. Em relação aos óbitos por COVID-19, o sexo masculino $\geq 80$ anos é o mais acometido, principalmente se houver a presença de uma ou mais comorbidades associadas. No quesito raça e cor, a cor parda ocupa o primeiro lugar tanto de casos confirmados quanto de óbitos. Os municípios mais atingidos foram os do interior do estado. CONCLUSÃO: É necessário fortalecer as medidas de enfrentamento à pandemia da COVID-19 no estado da Bahia, de forma a prevenir a ocorrência de novas transmissões e, consequentemente, aumento no número de casos e de óbitos por essa doença.

DESCRITORES: Coronavírus. Infecções por coronavírus. Estudo epidemiológico. Saúde pública.

\begin{abstract}
OBJECTIVE: To characterize the epidemiological profile of confirmed cases and deaths from COVID-19 in the state of Bahia. METHODS: Descriptive and retrospective epidemiological study based on secondary data extracted from the Epidemiological Bulletin of the State of Bahia, presented in tabular form with absolute and relative numbers. RESULTS: It was identified that most confirmed cases are female individuals in an age range of 30 to 39 years. Regarding COVID-19 deaths, males $\geq 80$ years old are the most affected, especially if one or more associated comorbidities are present. In terms of race and color, brown color ranks first in both confirmed cases and deaths. The municipalities most affected were those in the cities in the countryside. CONCLUSION: It is necessary to strengthen the measures to confront the pandemic of COVID-19 in the state of Bahia to prevent the occurrence of new transmissions and, consequently, an increase in the number of cases and deaths from this disease.
\end{abstract}

DESCRIPTORS: Coronavirus. Coronavirus Infections. Epidemiological study. Public Health. 


\section{Introdução}

O SARS-CoV-2 foi descoberto em dezembro de 2019 após casos registrados na cidade de Wuhan, China. O vírus faz parte da família Coronaviridae, a qual inclui outros vírus responsáveis por algumas infecções agudas graves como, por exemplo, a Síndrome Respiratória Aguda Grave e a Síndrome Respiratória do Oriente Médio¹. No Brasil, o primeiro caso foi confirmado em fevereiro de 2020 no estado de São Paulo e se tratava de um caso importado no qual o paciente havia retornado de viagem da Itália².

No estado da Bahia, o primeiro caso confirmado da doença foi no dia 06 de março de 2020, no município de Feira de Santana, sendo também um caso importado da Itália. A partir disso, até o dia 14 de janeiro de 2021, 1.581 .670 casos foram notificados, sendo 523.068 confirmados e 927.920 descartados por critérios laboratoriais (RT-PCR, imunológicos e teste rápidos). É importante destacar que, até o dia 14, 130.682 casos permaneciam em investigação epidemiológica.

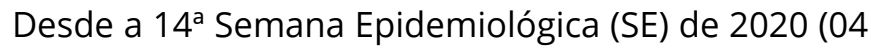
de abril de 2020) até a $1^{\text {a }}$ SE de 2021 (09 de janeiro de 2021), houve um aumento do número de casos confirmados de COVID-19 na Bahia, com incremento de $152.040,48 \%$ de casos nesse período 3 . Quanto aos óbitos, na $14^{\text {a }}$ SE foram notificadas 8 mortes. Já em 2021, na $1^{\text {a }} \mathrm{SE}$, o acumulado de óbitos chegou a 9.342, com uma média de 30 mortes por dia4.

A transmissão do vírus do SARS-CoV-2 é feita de pessoa para pessoa através de gotículas respiratórias produzidas durante a tosse, espirro e fala, além de objetos e superfícies contaminadas como celulares e maçaneta ${ }^{5}$. Alguns estudos sugerem a transmissão por pessoas assintomáticas ou levemente sintomáticas, dificultando mais ainda o controle do avanço da patologia ${ }^{6}$. A transmissão ainda está relacionada à propagação por meio de aglomerações, principalmente em ambientes familiares ${ }^{?}$.

Os sintomas da COVID-19 são variáveis, podendo se manifestar como um resfriado ou, nos casos mais graves, como uma pneumonia severa.
Dentreossintomasmais comuns, tem-seatosse, febre, coriza, dor de garganta e dificuldade para respirar $\stackrel{8}{\text {. }}$ Recentemente, pesquisas atentaram para a possibilidade da perda de olfato (anosmia) e do paladar (disgeusia) $)^{9}$ Sintomas gastrointestinais como diarreia, dor abdominal, náuseas e vômitos são atípicos, mas podem estar presentes ${ }^{10}$.

Apesar de a cura para o novo coronavírus ainda não ter sido encontrada, a busca por uma vacina segura e eficaz tem demonstrado resultados consistentes. Países como Israel, Emirados Árabes Unidos, Reino Unido e Estados Unidos da América apresentaram o plano de vacinação, dando início à imunização e gerando esperança na população mundial, que acompanha uma crescente no número de óbitos ${ }^{11}$.

Atualmente, o Brasil ocupa o $3^{\circ}$ lugar no ranking dos países com maior número de infectados pelo novo coronavírus. Já entre os estados brasileiros, a Bahia ocupa o $4^{\circ}$ lugar no ranking nacional, e o $1^{\circ}$ quando comparada a outros estados do nordeste ${ }^{12}$.

Nesse contexto, o Ministério da Saúde (MS) orienta a população sobre a importância da lavagem das mãos, distanciamento social e uso de máscaras como medidas de prevenção da transmissão do coronavírus ${ }^{5}$. Em conjunto com as orientações do MS, o Governo do Estado da Bahia suspendeu as aulas presenciais da rede de ensino estadual e privada, realizando testagem em estudantes, professores e servidores. Além disso, instituiu a suspensão de shows e eventos, higienização do transporte público e obrigatoriedade do uso de máscaras em ambientes públicos. Para viabilizar o atendimento remoto e monitoramento dos casos suspeitos ou confirmados de Covid-19, o Governo do Estado lançou o aplicativo Monitora Covid-19, que fornece orientações seguras, além de acompanhamento do estado de saúde do paciente ${ }^{13}$. Sendo assim, a pergunta que norteia esse estudo é: qual o perfil epidemiológico dos casos confirmados e óbitos por COVID-19 no estado da Bahia?

Frente à relevância da temática, o objetivo do estudo foi caracterizar o perfil epidemiológico dos casos confirmados e óbitos por COVID-19 no estado da Bahia. 


\section{Métodos}

Trata-se de um estudo epidemiológico descritivo retrospectivo, realizado a partir de dados secundários extraídos do Boletim Epidemiológico do Estado da Bahia, publicado no site da Secretaria de Saúde do Estado da Bahia (SESAB), disponível para consulta de forma online. Os dados foram coletados no dia 14 de janeiro de 2021. O Boletim englobava todas as informações referentes aos casos confirmados, descartados e óbitos desde o dia 27 de março de 2020.

Foram estudadas as variáveis: sexo (feminino/masculino), raça e cor e faixa etária (em anos), além das comorbidades associadas. Calculou-se o coeficiente de incidência dos cinco municípios com o maior número de casos no estado, e da capital baiana, dividindo o número de casos pela população de cada município e expresso por 10.000 habitantes.

Os dados foram tabulados e submetidos à análise estatística descritiva simples, utilizando o Microsoft Office Excel $365 ®$. Para os resultados, foram elaboradas tabelas de forma comparativa.

Este estudo não foi submetido ao Comitê de Ética em Pesquisa do Conselho Nacional de Saúde, já que os dados utilizados são secundários e estão disponíveis em uma plataforma de acesso livre e gratuito, conforme preconiza a Resolução n. 510, de 07 de abril de 2016, do Conselho Nacional de Saúde do Ministério da Saúde do Brasil. Dessa forma, todos os aspectos éticos da Resolução nº 466 de 12 de dezembro de 2012, do Conselho Nacional de Saúde do Ministério da Saúde do Brasil, foram respeitados.

\section{Resultados}

Desde o primeiro caso confirmado de COVID-19 no estado da Bahia no mês de março de 2020, o qual foi importado da Itália, 25 dias depois o estado já contava com 217 casos confirmados, 2 óbitos e 4.279 casos em investigação. Até o dia 14 de janeiro de 2021, foram confirmados 523.068 casos e 9.543 óbitos.

Em relação ao perfil dos pacientes com casos confirmados de COVID-19, houve uma predominância na faixa etária de 30-39 anos, sendo $24,36 \%$ dos casos. Porém, nos óbitos, a faixa etária com o maior percentual foi de $80+$ com 29,1\% (Tabela 1).

Tabela 1. Distribuição de casos e óbitos por COVID-19 segundo faixa etária. Bahia, Brasil, 2021

\begin{tabular}{|c|c|c|c|c|}
\hline \multirow[t]{2}{*}{ Faixa etária } & \multicolumn{2}{|c|}{ Casos } & \multicolumn{2}{|c|}{ Óbitos } \\
\hline & $\mathrm{n}$ & $\%$ & $\mathbf{n}$ & $\%$ \\
\hline$\leq 19$ & 58.544 & 11,22 & 72 & 0,7 \\
\hline $20-29$ & 92.958 & 17,77 & 109 & 1,1 \\
\hline $30-39$ & 127.435 & 24,36 & 328 & 3,4 \\
\hline $40-49$ & 102.990 & 19,69 & 708 & 7,4 \\
\hline $50-59$ & 68.031 & 13,01 & 1.187 & 12,4 \\
\hline $60-69$ & 38.908 & 7,44 & 2.019 & 21,2 \\
\hline $70-79$ & 20.698 & 3,96 & 2.341 & 24,5 \\
\hline $80+$ & 12.699 & 2,43 & 2.779 & 29,1 \\
\hline Ignorado & 805 & 0,15 & 0 & 0,00 \\
\hline Total & 523.068 & $100 \%$ & 9.543 & $100 \%$ \\
\hline
\end{tabular}


Já relacionado ao sexo, houve uma predominância do sexo feminino nos casos confirmados (54,63\%) quando comparado ao sexo masculino (45,19\%), sendo que $0,19 \%$ dos casos não tinham informação. Em contrapartida, o número de óbitos foi maior no sexo masculino com 56,52\% das mortes (Tabela 2).

Tabela 2. Distribuição de casos e óbitos por COVID-19 por sexo. Bahia, Brasil, 2021

\begin{tabular}{lccc}
\hline & Sexo & Casos confirmados & Óbitos \\
& $\%$ & $n$ & $43,48 \%$ \\
\hline Feminino & $54,63 \%$ & 4.149 & $56,52 \%$ \\
Masculino & $45,19 \%$ & 5.394 & $0,00 \%$ \\
Sem Informação & $0,19 \%$ & 0 & $100 \%$ \\
\hline Total & $100 \%$ & 9.543 & \\
\hline Fonte: Boletim Epidemiológico do Estado da Bahia - SESAB (2021) & &
\end{tabular}

Em relação ao quesito raça/cor, foi constatado que $50,60 \%$ são de cor parda, $12,22 \%$ brancos e $8,21 \%$ pretos. No que diz respeito aos óbitos, a cor parda também ocupa o primeiro lugar, representando $55,11 \%$ do número total (Tabela 3).

Tabela 3. Distribuição de casos e óbitos por COVID-19 segundo raça e cor. Bahia, Brasil, 2021

\begin{tabular}{lcccc}
\hline Raça/ cor & \multicolumn{2}{c}{ Casos } & \multicolumn{2}{c}{ Óbitos } \\
& $n$ & $\%$ & $n$ & 55,11 \\
\hline Parda & 264.684 & 50,60 & 5.259 & 0,65 \\
Amarela & 82.334 & 15,74 & 62 & 19,26 \\
Branca & 63.915 & 12,22 & 1.838 & 1.402 \\
Preta & 42.962 & 8,21 & 12 & 0,13 \\
Indígena & 1.817 & 0,35 & 970 & 10,16 \\
Sem informação & 67.356 & 12,88 & 9.543 & $100 \%$ \\
\hline Total & 523.068 & $100 \%$ & &
\end{tabular}

O percentual de casos com comorbidades foi de 70,94\%, havendo uma maior ocorrência de hipertensão arterial sistêmica (33,58\%), seguido de diabetes mellitus (30,21\%), sendo que 20,16\% não tinham informações (Tabela 4). 
Tabela 4. Distribuição de óbitos por COVID-19 segundo comorbidades. Bahia, Brasil, 2021

\begin{tabular}{|c|c|c|}
\hline \multirow[t]{2}{*}{ Comorbidades } & \multicolumn{2}{|c|}{ Óbitos } \\
\hline & $\mathbf{n}$ & $\%$ \\
\hline Hipertensão arterial sistêmica & 3.205 & 33,58 \\
\hline Diabetes Mellitus & 2.883 & 30,21 \\
\hline Sem informação & 1.924 & 20,16 \\
\hline Doença cardiovascular exceto HAS & 1.765 & 18,49 \\
\hline Sem comorbidade & 842 & 8,82 \\
\hline Doença renal crônica & 703 & 7,36 \\
\hline Obesidade & 540 & 5,66 \\
\hline Doença respiratória crônica & 474 & 4,97 \\
\hline Neoplasias & 362 & 3,79 \\
\hline Doença do Sistema Nervoso & 227 & 2,38 \\
\hline Demências (incluindo Alzheimer) & 150 & 1,57 \\
\hline Tabagismo & 123 & 1,29 \\
\hline Imunodeficiências & 112 & 1,17 \\
\hline Doenças hematológicas & 69 & 0,72 \\
\hline Doença hepática & 68 & 0,71 \\
\hline Doença endócrina, nutricional e metabólica (exceto obesidade e DM) & 61 & 0,64 \\
\hline Outros & 48 & 0,50 \\
\hline Glaucoma e osteoartrose & 30 & 0,31 \\
\hline
\end{tabular}

Em relação a capital Salvador, o número de casos foi de 116.582, representando uma incidência de 11,65/10.000 habitantes. Os cinco municípios com maior incidência de contaminação foram: Ibirataia (1.058,64/10.0000), Muniz Ferreira (869,03/ 10.0000), Conceição do Coité $(856,9 / 10.0000)$, Itabuna $(823,41 / 10.0000)$ e Jucuruçu (817,44/10.0000). A partir do dia 13 de agosto de 2020, os dados referentes aos óbitos por município de residência deixaram de ser disponibilizados no Boletim Epidemiológico.

\section{Discussão}

Os números de casos e a crescente expansão da COVID-19 no cenário mundial tem causado preocupação. No Brasil, o número de infectados é de 8.455.059, com 209.296 óbitos segundo atualização do Painel COVID-19 do Ministério da Saúde. Esse crescimento afetou também o estado da Bahia, que hoje ocupa a $4^{a}$ posição no ranking nacional de casos confirmados ${ }^{12}$.

No dia 14 de janeiro de 2021, o número de casos confirmados no estado da Bahia foi de 4.113, correspondendo a uma taxa de crescimento de $0,8 \%$ quando comparado ao dia anterior, o maior número desde o dia 20 de dezembro de 2020. Já em relação aos óbitos, a média era de 30 por dia, significando uma estabilidade de $3 \%$ no número de mortes. É importante ressaltar que os números de óbitos registrados no Boletim Epidemiológico são de acordo com a data do falecimento, não representando necessariamente as mortes ocorridas no mês estudado 4 .

Dentre os pacientes com casos confirmados de COVID-19 no estado da Bahia, 24,36\% eram referentes a faixa etária de 30-39 anos, diferente da cidade mais populosa da China, Xangai, que entre janeiro e fevereiro de 2020, $47,4 \%$ dos pacientes admitidos possuía a média de 51 anos ${ }^{14}$. Já em relação aos óbitos, a faixa etária mais acometida é de $\geq 80$ anos, o que corrobora com os achados em um estudo da região norte do Brasil 15 e se assemelha com os dados disponibilizados pela Secretaria de Saúde do Estado de São Paulo, líder no ranking nacional dos estados mais atingidos pelo novo coronavírus ${ }^{16}$. 
No que se refere ao sexo, entre os casos confirmados registrados no Boletim Epidemiológico do Estado da Bahia do dia 14 de janeiro, o sexo feminino apresentou uma maior frequência. Esses achados corroboram com um estudo feito no Maranhão $\frac{17}{17}$ mas diferenciam-se dos resultados encontrados em uma revisão sistemática e metanálise realizada com estudos da China, onde $60 \%$ dos infectados eram do sexo masculino ${ }^{18}$. Pressupõe-se que isso se dá pelo fato de as mulheres acessarem mais os cuidados de saúde do que os homens ${ }^{19}$.

Quanto aos óbitos, o número foi maior no sexo masculino. Isso também pode ser explicado pela baixa procura do público masculino pelos serviços de saúde, bem como por um viés biológico, onde um estudo demonstrou que o cromossomo $X$ contém uma maior densidade de genes relacionados à imunidade. Além disso, o estrogênio, que é um hormônio sexual feminino, atua como um ativador imunológico, contribuindo para uma resposta imunológica vigorosa ${ }^{19}$.

No tocante à raça/cor, tanto o número de casos da infecção quanto o número de óbitos foram maiores na cor parda. A Pesquisa Nacional por Amostra de Domicílios Contínua trimestral publicada pelo Instituto Brasileiro de Geografia e Estatística (IBGE) referente ao $1^{\circ}$ trimestre de 2020 traz que 58,5\% da população baiana é parda, enquanto os pretos correspondem a $22,5 \%$ e os brancos a $17,9 \%{ }^{20}$. Vale destacar que os 67.356 casos que não tinham informações podem gerar distorção da análise desta variável.

Uma análise feita pelo Núcleo de Operações e Inteligência em Saúde (NOIS) da PUC Rio traz a comparação percentual entre os casos de óbitos e recuperados no quesito raça/cor. Segundo os dados, o número de negros que vieram a óbito foi superior ao número de brancos ${ }^{21}$. Os achados evidenciam as desigualdades sociais e diferenças no acesso à saúde, onde, apesar de o Sistema Único de Saúde (SUS) ser universal, atende principalmente a parcela mais carente da população brasileira. Apesar disso, o SUS continua sendo sucateado por consequência da lógica capitalista que prioriza o setor privado, reduzindo a qualidade dos serviços prestados 22 .

Outro ponto importante é o número de casos sem informação que, caso fossem identificados, poderiam contribuir e enriquecer análises a respeito da dimensão racial envolvida na pandemia do novo Coronavírus.
Em relação às comorbidades associadas aos óbitos, a hipertensão e diabetes ficaram entre as principais causas, coadunando com os achados de uma metanálise realizada na China, que mostrou um risco aumentado de agravamento em indivíduos com hipertensão e diabetes ${ }^{23}$. Um estudo publicado no periódico Archives of Internal Medicine da Sociedade Polonesa de Medicina Interna trouxe que a hipertensão está relacionada a um risco de quase 2,5 vezes maior para o desenvolvimento da forma grave da COVID-19, assim como com um maior risco de mortalidade ${ }^{24}$. É importante ressaltar que os números de casos relativos às comorbidades não refletem o número total de óbitos, visto que um só paciente pode apresentar várias comorbidades associadas.

Os municípios com maior incidência de contaminação por 10.000 habitantes no estado foram Ibirataia, Muniz Ferreira, Conceição do Coité, Itabuna e Jucuruçu. Esses dados demonstram a ascensão de um novo fenômeno de interiorização da pandemia, onde os municípios de menor porte tendem a notificar mais casos da doença. Nesse sentido, a Fiocruz, por meio de uma nota técnica, expressou preocupação em relação à ocupação dos leitos de UTI que pode contribuir para a sobrecarga do sistema de saúde ${ }^{25}$. Vale destacar que até 14 de janeiro de 2021, existiam 2.031 leitos, sendo que 725 leitos de UTI e 547 leitos clínicos estavam ocupados por COVID-19 no estado da Bahia.

Compreendendo que os municípios com maior taxa de contaminação correspondem a cidades do interior, espera-se que a taxa de letalidade seja um pouco mais elevada em função do número total de habitantes. Além disso, por se tratarem de municípios de pequeno porte, o acesso à assistência especializada depende da disponibilidade de leitos nas cidades maiores que também são limitados, gerando a sobrecarga do sistema de saúde. Em um contexto geral, o coeficiente de letalidade do estado da Bahia gira em torno de $1,82 \%$.

Além disso, o estado realizou mais de 100.000 testes para detecção da COVID-19 sendo que, até 14 de janeiro de 2021, o número de recuperados (505.398) encontrava-se maior que o número de casos ativos (8.127), o que significa que apesar do alto índice de contaminação, 96,62\% dos casos confirmados se recuperaram em isolamento domiciliar ou internamento hospitalar em casos de agravo 3 . 
Com base nos dados apresentados no estudo, faz-se necessário intensificar medidas de controle da disseminação do vírus, com estratégias de prevenção visando diminuir o número de casos e óbitos. A lavagem das mãos é uma das principais formas de evitar a transmissão do vírus quando feita de forma correta e com frequência. Recomenda-se a utilização de máscara em todos os ambientes como uma barreira física contra a saída de gotículas potencialmente contaminadas. É necessário ainda manter o distanciamento social, a fim de evitar aglomerações e a contaminação em massa. Outros cuidados como cobrir nariz e boca ao tossir ou espirrar, evitar o compartilhamento de objetos pessoais e não ter contato com outras pessoas em caso de suspeita ou confirmação da doença, constituem estratégias essenciais para redução do risco de transmissão do SARS-CoV- $2^{5}$.

Este estudo possui limitações relativas à atualização diária do Boletim Epidemiológico do Estado da Bahia, publicado pela Secretaria do Estado da Bahia, bem como aos possíveis casos e óbitos subnotificados, principalmente àqueles assintomáticos ou menos sintomáticos. Dessa forma, é necessário o desenvolvimento de novos estudos que retratem as mudanças do perfil epidemiológico no estado para embasar Políticas de Saúde Pública referentes ao atual contexto da pandemia.

\section{Conclusão}

Diante do exposto, percebe-se um crescente no número de casos confirmados pelo novo Coronavírus na Bahia, expandindo-se para cidades do interior do estado, o que exige um maior combate à disseminação do vírus. O levantamento apontou ainda que a maioria dos infectados são indivíduos do sexo feminino entre 30 a 39 anos de raça/cor parda. Em relação aos óbitos, os mais atingidos são os indivíduos do sexo masculino com 80 anos ou mais de raça/cor parda, seguido por brancos e pretos. Foi possível perceber que a presença de uma ou mais comorbidades associadas contribuiu para um pior prognóstico.

Por conseguinte, é necessário fortalecer as medidas de enfrentamento à pandemia a fim de prevenir a ocorrência de novas transmissões, aumento do número de casos e, consequentemente, aumento do número de óbitos.

\section{Contribuições dos autores}

Machado AG participou da concepção, delineamento, coleta, análise e interpretação de dados da pesquisa, redação, revisão crítica e relevante do conteúdo do artigo científico. Batista MS participou da concepção, delineamento, coleta, análise e interpretação de dados da pesquisa, redação, revisão crítica e relevante do conteúdo intelectual e aprovação final da versão a ser publicada. Souza MC participou da redação do artigo científico, revisão crítica do conteúdo intelectual e aprovação final da versão a ser publicada.

\section{Conflitos de interesses}

Nenhum conflito financeiro, legal ou político envolvendo terceiros (governo, empresas e fundações privadas, etc.) foi declarado para nenhum aspecto do trabalho submetido (incluindo, mas não se limitando a subvenções e financiamentos, participação em conselho consultivo, desenho de estudo, preparação de manuscrito, análise estatística, etc.).

\section{Referências}

1. World Health Organization. Origin of SARS-CoV-2 [Internet]. Geneva: World Health Organization; 2020. Disponível em: https:// apps.who.int/iris/bitstream/handle/10665/332197/WHO-2019nCoV-FAQ-Virus_origin-2020.1-eng.pdf

2. Ministério da Saúde (Brasil). Brasil confirma o primeiro caso da doença [Internet]. Brasil: Ministério da Saúde; 2020. Disponível em: https://www.gov.br/saude/pt-br/assuntos/noticias/brasilconfirma-primeiro-caso-de-novo-coronavirus

3. Secretaria de Saúde (Brasil). Boletim Epidemiológico Bahia - Covid-19 [Internet]. Bahia: Secretaria de Saúde do Estado da Bahia; 2020. Disponível em: http://www.saude.ba.gov. br/wp-content/uploads/2021/01/BOLETIM_ELETRONICO_ BAHIAN 296 14012021.pdf

4. Secretaria de Saúde (Brasil). Boletim Infográfico Bahia Covid-19 [Internet]. Bahia: Secretaria de Saúde do Estado da Bahia; 2021. Disponível em: http://www.saude.ba.gov.br/wpcontent/uploads/2021/01/Boletim-Infografico-14-01-2021.pdf

5. Ministério da Saúde (Brasil). CORONAVÍRUS (COVID-19) [Internet]. 2020. [citado 2020 jun 23]. Disponível em: https:// coronavirus.saude.gov.br/sobre-a-doenca\#transmissao

6. Bittencourt RJ. Testagem de rastreio e busca ativa de infectados assintomáticos pelo SARS-COV-2: a visão do planejamento em saúde pública. Com Ciências Saúde [Internet]. 2020;31(supl 1):816. Disponível em: http://www.escs.edu.br/revistaccs/index.php/ comunicacaoemcienciasdasaude/article/view/649/288 
7. Ferreira EMS, Souza BG, Silva PWP, Miranda WL, Pimenta RS, Silva JF. Sars-cov-2-aspectos relacionados a biologia, propagação e transmissão da doença emergente Covid-19. Revista Desafios [Internet]. 2020;7-13. Disponível em: https://sistemas.uft. edu.br/periodicos/index.php/desafios/article/view/8859/16714

8. Araújo ACA, Amaral JV, Sousa JN, Fonseca MCS, Viana CMC, Mendes PHM, et al. Covid-19: Análise de casos confirmados em Teresina, Piauí, Brasil. Rev Pre Infec e Saúde. 2020;6:10569. https://doi.org/10.26694/repis.v6i0.10569

9. Lapostolle F, Schneider E, Vianu I, Dollet G, Roche B, Berdah J, et al. Clinical features of 1487 COVID-19 patients with outpatient management in the Greater Paris: the COVID-call study. Intern Emerg Med. 2020;15:813-7. https://doi.org/10.1007/s11739-020$\underline{02379-z}$

10. Agência Nacional de Vigilância Sanitária (Brasil). Nota técnica n. 04/2020 GVIMS/GGTES. Orientações para serviços de saúde: medidas de prevenção e controle que devem ser adotadas durante a assistência aos casos suspeitos ou confirmados de infecção pelo novo coronavírus (SARS-CoV-2) [Internet]. Brasil: Agência Nacional de Vigilância Sanitária; 2020. [citado 2021 fev 15]. Disponível em: https://www.telessaude.unifesp.br/images/ downloads/Nota\%20Te\%CC\%81 cnica\%20n\%2004-2020\%20 GVIMS-GGTES-ANVISA-ATUALIZADA.pdf

11. BBC News Brasil. Vacina contra Covid: os países que lideram o ranking de imunização no mundo [Internet]. Brasil: BBC News Brasil; 2021. [citado 2021 jan 17]. Disponível em: https://www.bbc. com/portuguese/internacional-55550798

12. Coronavírus Brasil. Painel Coronavírus [Internet]. Brasil: Ministério da Saúde; 2021. [citado 2021 jan 17]. Disponível em: https://covid.saude.gov.br/

13. Governo do Estado da Bahia. Entenda as medidas de combate ao coronavírus na Bahia [Internet]. 2020. [citado $2021 \mathrm{fev} \mathrm{16].}$ Disponível em: http://www.bahia.ba.gov.br/2020/08/noticias/ saude/entenda-as-medidas-de-combate-ao-coronavirus-na-bahia/

14. Shen Y, Zheng F, Sun D, Ling Y, Chen J, Li F, et al. Epidemiology and clinical course of COVID-19 in Shanghai, China. Emerg Microbes Infect. 2020;9(1):1537-45. https://doi.org/10.1080/22221 751.2020.1787103

15. Mendonça FD, Rocha SS, Pinheiro DLP, Oliveira SV. Região Norte do Brasil e a pandemia de COVID-19: análise socioeconômica e epidemiológica. J. Health NPEPS [Internet]. 2020;5(1):20-37. Disponível em: https://periodicos.unemat.br/ index.php/jhnpeps/article/view/4535

16. Novo Coronavírus (COVID-19) - Situação Epidemiológica [Internet]. São Paulo. 2020 [citado 2020 jul 01]. Disponível em: https://www.prefeitura.sp.gov.br/cidade/secretarias/saude/ vigilancia_em_saude/doencas_e_agravos/coronavirus/index. php?p=295572
17. Almeida JS, Cardoso JA, Cordeiro EC, Lemos M, Araújo TME, Sardinha AHL. Caracterização epidemiológica dos casos de covid-19 no maranhão: Uma breve análise. Scielo Preprints. 2020;6:10477. https://doi.org/10.1590/SciELOPreprints.314

18. Khalili M, Karamouzian M, Nasiri N, Javadi S, Mirzazadeh A, Sharifi H. Epidemiological characteristics of COVID-19: a systematic review and meta-analysis. Epidemiol Infect. 2020;148:e130. https://dx.doi. org/10.1017\%2FS0950268820001430

19. Schurz H, Salie M, Tromp G, Hoal EG, Kinnear CJ, Möller $M$. The $X$ chromosome and sex-specific effects in infectious disease susceptibility. Hum Genomics. 2019;13(1):2. https://doi. org/10.1186/s40246-018-0185-z

20. Instituto Brasileiro de Geografia e Estatística. Pesquisa Nacional por Amostra de Domicílios Contínua trimestral [Internet]. Brasil: Instituto Brasileiro de Geografia e Estatística; 2020. [citado 2021 jan 17]. Disponível em: https://sidra.ibge.gov. br/tabela/6403\#resultado

21. Núcleo de Operações e inteligência em Saúde (Brasil). Nota Técnica 11: Análise socioeconômica da taxa de letalidade da COVID-19 no Brasil [Internet]. 2020. [citado $2021 \mathrm{fev} \mathrm{16].}$ Disponível em: https://ponte.org/wp-content/uploads/2020/05/ NT11-An\%C3\%A1lise-descritiva-dos-casos-de-COVID-19.pdf

22. Araujo EM, Caldwell KL, Santos MPA, Souza IM, Rosa PLFS, Santos ABS, et al. COVID-19 morbimortality by race/skin color/ ethnicity: the experience of Brazil and the United States / Covid-19 - Morbimortalidade pela COVID-19 segundo raça/cor/etnia: a experiência do Brasil e dos Estados Unidos. SciELO Preprints. 2020. https://doi.org/10.1590/SciELOPreprints.1318

23. Wang B, Li R, Lu Z, Huang Y. Does comorbidity increase the risk of patients with COVID-19: evidence from meta-analysis. Aging (Albany NY). 2020;12(7):6049-57. https://doi.org/10.18632/ aging. 103000

24. Lippi G, Wong J, Henry BM. Hypertension in patients with coronavirus disease 2019 (COVID-19): a pooled analysis. Pol Arch Intern Med. 2020;130(4):304-9. https://doi.org/10.20452/ pamw.15272

25. Fundação Oswaldo Cruz. Tendências atuais da pandemia de Covid-19: Interiorização e aceleração da transmissão em alguns estados [Internet]. 2020. [citado 2020 jul 01]. Disponível em: https://portal.fiocruz.br/sites/portal.fiocruz.br/files/documentos/ monitoracovid_notatecnica_28_04.pdf 